\title{
Self-consistent models of triaxial galaxies in MOND gravity
}

\author{
You-gang Wang ${ }^{1,3}$, Xufen $\mathrm{Wu}^{2}$, HongSheng Zhao ${ }^{1,2}$ \\ hz4@st-andrews.ac.uk
}

\begin{abstract}
The Bekenstein-Milgrom gravity theory with a modified Poisson equation is tested here for the existence of triaxial equilibrium solutions. Using the nonnegative least square method, we show that self-consistent triaxial galaxies exist for baryonic models with a mild density cusp $\rho \sim \frac{\Sigma}{r}$. Self-consistency is achieved for a wide range of central concentrations, $\Sigma \sim 10-1000 \mathrm{M}_{\odot} \mathrm{pc}^{-2}$, representing low-to-high surface brightness galaxies. Our results demonstrate for the first time that the orbit superposition technique is fruitful for constructing galaxy models beyond Newtonian gravity, and triaxial cuspy galaxies might exist without the help of Cold dark Matter.
\end{abstract}

Subject headings: galaxies: kinematics and dynamics- methods: numerical - dark matter

\section{Introduction}

Constructing models of galaxies in triaxial equilibrium is a classical challenge in dynamics, either in Newtonian or Modified gravity. However, due to numerical constructions, few such models have been developed in Newtonian since the pioneering work of Schwarzchild(1979) on triaxial elliptical galaxies, and Zhao (1996) on the fast rotating triaxial bar of the Milky Way. Merritt \& Fridman (1996) (hereafter MF96) considered a model with a central density cusp $r^{-1}$ and found that it is possible to construct a model of triaxial equilibrium galaxies self-consistently in Newtonian dynamics. Models with a steeper density cusp are not in equilibrium. This has generated interests in understanding cusp-triaxiality relation, the triaxiality-velocity anisotropy relation and the cusp-black hole relation. Recently, Capuzzo-Dolcetta et al. (2007) modeled $r^{-1}$ cuspy triaxial galaxies in $\Lambda$ Cold Dark

\footnotetext{
${ }^{1}$ National Astronomical Observatories, Chinese Academy of Sciences, Beijing 100012

2 SUPA, School of Physics and Astronomy, University of St Andrews, KY16 9SS, UK

${ }^{3}$ Department of Astronomy Peking University, Beijing 100871, China
} 
Matter (CDM) haloes. They took the model of MF96 as the luminous density distribution, and found that a model of cupsy triaxial galaxies with CDM halos is also self-consistent.

A tough problem for $\Lambda \mathrm{CDM}$ ( $\Lambda$ plus Cold Dark Matter) is that there is no physics to justify $\Lambda$ the tiny cosmological constant or dark energy (see White 2007, Sarkar 2007). Although future particle physics experiments might well prove the existence of new species of particles and vacuum energy, their experimental-determined abundance could easily be factors of a few different from the 1:3 ratio as precisely required by fitting observations of Microwave background (Zhao 2006). This motivates exploring alternative theories like the co-variant version of Modified Newtonian Dynamics (MOND) in the mean-time. E.g., instead of more traditional interpretation of modified gravity (Bekenstein 2004), MOND was given the interpretation as a co-variant Dark Energy (DE) the $\mathbf{V} \boldsymbol{\Lambda}$ model of Zhao (2007). This model uses General Relativity plus a non-uniform Dark Energy (DE) fluid (described by a four-vector flow) to give both the effects of DE and DM. Perturbations of such DE fluid bends space-time as an effective DM without actually invoking DM. Unlike $\Lambda$ CDM halos, which are positive and nearly round, the effective DM can sometimes be extremely flattened or negative in some regions of galaxies or clusters (Wu et al. 2007, 2008), although the data are not good enough to tell such negative regions yet (Nipoti et al. 2007a).

Overall MOND and $\Lambda$ CDM halos are comparably successful in explaining the flat rotation curves of high surface brightness discs and satellites orbits at large radii (e.g., Angus et al. 2008). However, both theories are faced with severe challenges: $\Lambda$ CDM hinges on uncertain feedback on galactic scales, and even the maximum feedback falls short of explaining the velocity curves of low-surface brightness systems (Gnedin \& Zhao 2002); Bekenstein's (2004) co-variant MOND seems to rely on non-relativistic neutrinos on large scale to match the weak lensing data (Angus et al. 2007) and the Cosmic Background Radiation (Skordis et al. 2006).

The tight correlation observed between the mass profiles of baryonic matter and dark matter at all radii in spiral galaxies (e.g., McGaugh et al. 2007; Famaey et al. 2007) is still the best case for the MOND paradigm of Milgrom (1983), which postulates that for accelerations below $a_{0}=1.2 \times 10^{-10} \mathrm{~m} \mathrm{~s}^{-2}$ the effective gravitational attraction approaches $\left(g_{N} a_{0}\right)^{1 / 2}$, where $g_{N}$ is the usual Newtonian gravitational field. Indeed, without resorting to galactic dark matter, this simple prescription is amazingly successful at reproducing galactic rotation curves over five decades in mass ranging from tiny dwarfs (e.g., Gentile et al. 2007) to early-type disk galaxies (e.g., Sanders \& Noordermeer 2007) to massive ellipticals (Milgrom \& Sanders 2003). Some outliers exist in models of gravitational lensing (Zhao et al. 2006,

\footnotetext{
${ }^{1}$ although these problems appear resolvable in the V $\mathbf{\Lambda}$ model (Zhao 2007).
} 
Chen \& Zhao 2006, Shan et al. 2008). Lensing and structure formation are well-defined calculations in co-variant MOND (Halle \& Zhao 2007). Galaxy formation simulations in classical MOND have been carried out too, and it is possible to form bars (Tiret \& Combes 2007), and elliptical galaxies by mergers (Nipoti et al. 2007b), and spherical bulges by instability (Zhao, Xu \& Dobbs 2008).

Very little is known about the triaxial stationary equilibrium in non-Newtonian dynamics, although one speculates that such equilibrium might exist. In this paper, we test the existence of triaxial galaxies in the Bekenstein-Milgrom MOND theory, a well-defined classical theory with relativisitic and cosmological extentions. We apply the same density model given by MF96 with a fixed mass, and axis ratios. Our aim is to examine whether this model is self-consistent in MOND.

The MOND theory is scale-dependent. When the gravitational acceleration is below $a_{0}$, the scaling deviates from the $r^{-2}$ Newtonian law. Here we consider models with different

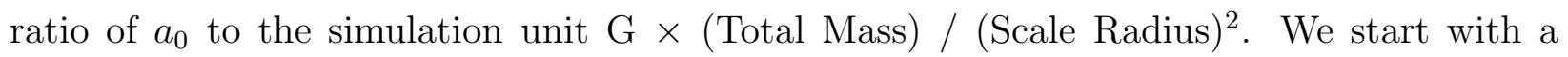
nearly Newtonian system (high surface brightness) and finish with a deep-MOND system (low surface brightness).

The rest of the paper is organized as follows. In $\S 2$, we present the density distribution of the galaxy and the corresponding potential and forces. The orbits are calculated in $\S 3$. The construction of self-consistent models is discussed in $\S 4$, and in $\S 5$, we give our conclusions and disscussions.

\section{The density, potential distribution and forces in the systems of triaxial galaxies}

The baryon component adopted by Capuzzo-Dolcetta (2007) has the following ellipsoidal mass distribution:

$$
\rho_{b}(R)=\frac{M}{2 \pi h_{x} h_{y} h_{z}} \frac{1}{R(1+R)^{3}},
$$

where $R^{2}=\frac{x^{2}}{h_{x}^{2}}+\frac{y^{2}}{h_{y}^{2}}+\frac{z^{2}}{h_{z}^{2}}\left(h_{z} \leq h_{y} \leq h_{x}\right)$ is the length of the major axis, and $M$ is the total baryon mass. For the ratio of the three major axes, we adopt $h_{x}: h_{y}: h_{z}=1: 0.86: 0.7$ throughout this paper. This density profile was studied in MF96, but in their work, it represented the density profile of the dark matter. The density profile is plotted in the top panel of Fig. 1, Density is cuspy and scales as $R^{-1}$ at the center and decreases quickly as $R^{-4}$ at large radii.

Hereafter, we adopt units in which the total mass $M$, the x-axis scale length $h_{x}$, and 
the gravitational constant $G$ are unity. Thus one unit of time corresponds to

$$
\begin{aligned}
T & =G^{-1 / 2} h_{x}^{3 / 2} M^{-1 / 2} \\
& =1.49 \times 10^{6} \mathrm{yr}\left(\frac{M}{10^{11} M_{\odot}}\right)^{-1 / 2}\left(\frac{h_{x}}{1 \mathrm{kpc}}\right)^{3 / 2}
\end{aligned}
$$

The MOND nonrelativistic field equation is different from Poisson's equation in Newton's theory (Bekenstein \& Milgrom 1984), and it has the following form

$$
\begin{aligned}
4 \pi G \rho & =\nabla \cdot(\mu \nabla \Phi) \\
& =\partial_{x}\left(\mu \partial_{x} \Phi\right)+\partial_{y}\left(\mu \partial_{y} \Phi\right)+\partial_{z}\left(\mu \partial_{z} \Phi\right),
\end{aligned}
$$

where $\Phi$ is the gravitational potential generated by the density distribution $\rho$. We adopt the function $\mu$ as in Zhao \& Famaey (2006), which is given by

$$
\mu(g)=\frac{g}{g+a_{0}},
$$

Clearly $\mu=1$ for $g \rightarrow \infty$ and $\mu=g / a_{0}$ for $g \rightarrow 0$, as expected for any MOND theory. The gravity strength $g$ is given by

$$
\begin{aligned}
g & =|\nabla \Phi| \\
& =\sqrt{\left(\partial_{x} \Phi\right)^{2}+\left(\partial_{y} \Phi\right)^{2}+\left(\partial_{z} \Phi\right)^{2}} .
\end{aligned}
$$

From equation (5) it can be seen that the equation (3) does not reduce to Poisson's equation if $a_{0}$ is not small enough. We solve equation (3) numerically, using the Bologna group's MOND Poisson Solver (Ciotti et al. 2006), which is based on a spherical grid. This code yields results which are consistent with that of the Cartesian grid code of the Paris group (Tiret \& Combes 2007). We apply the Poisson Solver with a resolution of $256 \times 64 \times 128$ grids points, and choose the radial grid points as $r_{i}=2.0 \tan \left[(i+0.5) \frac{0.5 \pi}{256+1}\right] \mathrm{kpc}$. We obtain the components of gravity in $\mathrm{x}, \mathrm{y}, \mathrm{z}$ directions, ie. values of $\partial_{x}, \partial_{y}$, and $\partial_{z}$ from the Poisson Solver.

In the lower panel of Figure 1, we show the potential radial profile along the major axis for different values of $a_{0}$ in simulation units. In the middle panel we show the radial gravity $\left|g_{r}\right| / a_{0}$ as a function of $R$ along the long axis. In the simulations $a_{0}$ is dimensionless and is equal to $0.0833,0.833$ and 8.33 , which correspond to simulation units of $m=1.0 \times 10^{10} M_{\odot}$, $1.0 \times 10^{9} M_{\odot}$, and $1.0 \times 10^{8} M_{\odot}$ respectively, and $G=1$. In all simulations, we start with a total mass of $M=10 \mathrm{~m}$ (See Table 1). We find that there is no obvious difference between the mild and weak gravity cases, except in the central region. For the strong gravity case $\left(a_{0}=0.083\right)$, the gravitational potential falls quickly at the outer regions of the galaxy. 
The left panel of Figure 2 shows the isopotentials (solid lines) in the gas with axial ratios $h_{x}: h_{y}: h_{z}=1: 0.86: 0.7$ at five different radii, $R=2,10,20,30$ and $50 \mathrm{kpc}$. There are no obvious differences with different values of $a_{0}$ (see Fig. $2 \mathrm{~b}$ ). Further more, comparing with the density contours (the dotted lines on the Fig. 2a), the isopotential contours are more spherical than the isodensity (Fig. 2b), which is expected (see also the examples in Lee \& Suto (2003) and Wang \& Fan (2004)). The three-dimensional potential distribution is approximately ellipsoidal, with eccentricity increasing from the center to the outer part of the cluster. For example, the axial ratio of potential distribution is $h_{z}^{p}: h_{x}^{p}=0.90: 1.0$ at $2 \mathrm{kpc}$, while $h_{z}^{p}: h_{x}^{p}=0.99: 1.0$ at $100 \mathrm{kpc}$. The dashed lines are isodensities of the effective DM halo in MOND. On the Fig. 2b, it is interesting that the axis ratio of effective DM densities are smaller than that of baryon densities at radii smaller than $1 \mathrm{kpc}$, where baryons dominate the dynamics. To produce the same dynamical behavior(e.g. the rotation curves of galaxies), the CDM halos always adopt a constant axis ratio of dark matter density in the inner and outer part of galaxies, otherwise the CDM models would be much too complex.

For the model of the elliptical galaxy, one important parameter is the effective radii. Hernquist (1990) shew that the effective of the elliptical galaxy is $\sim 1.8153 h_{x}$ for a spherical system. In our models, the effective radii is also $\sim 1.8 h_{x}$ because this parameter is independent of gravity and is set by the baryon density profile.

MOND is equivalent to an effective DM theory. The effective density can be obtained from

$$
4 \pi G \rho_{D M}^{e f f}=\nabla \cdot[(1-\mu) \nabla \Phi] .
$$

This density can be quite different from both the baryonic density and also the nearly ellipsoidal density of the CDM halo. A example is shown in Fig. 2.

\section{Integration of orbits}

In Newton's theory, energy, and the three components of the angular momentum, are integrals of motion in a spherical system. However, only energy is kept as a constant in the triaxial potential. MOND does not change the number of constants of motion. BekensteinMilgrom's theory conserves energy for a stationary potential. In order to obtain a specified accuracy, we use the $7 / 8$ order Runge-Kutta algorithm (Fehlberg 1968) to carry out the integrations. The integration time for a full orbit is 100 dynamical times. One dynamical time is defined as the period of the 1:1 resonant orbit in the $\mathrm{x}-\mathrm{y}$ plane. Table 2 lists the periods of $1: 1$ resonant orbits in the $x-y$ plane as a function of energy. 
In order to produce a library of all possible orbits, we followed Schwarzschild (1993) and MF96 in selecting our initial conditions. Specifically, the definition of start-space is the same as in MF96. The orbital catalogues include both initially stationary orbits and $\mathrm{x}-\mathrm{Z}$

plane launched orbits with nonzero velocity $v_{y}$, where $v_{y}=\sqrt{2(E-\Phi)} \neq 0$ is the velocity along the y-axis. For each model we have 20 energy surfaces. The surfaces are the critical surface of the shells which divide the total system into 21 parts of equal mass. One energy consists 342 initial conditions, where 192 orbits are from the stationary start-space and the rest are from the $\mathrm{x}-\mathrm{z}$ start-space. Therefore, for each model we have 6840 orbits.

Figure 3 shows the orbits in three separate planes for MOND models. The energies of orbits are from shell 10. The orbits in a triaxial MOND potential are similar to the orbits in a triaixal Newtonian potential, which yield the box, tube and stochastic orbits.

\section{Construction of self-consistent models}

Since Schwarzschild (1979) proposed a numerical method to construct self-consistent models of galaxies, orbit superposition techniques have become an important tool in dynamical modelling (e.g., Rix 1997; van der Marel et al. 1998; Kuijken 2004; Binney 2005; van de Ven 2006; Capuzzo-Dolcetta et al. 2006). The procedure of constructing such models can be described as follows:

(1)A three-dimensional mass distribution is employed and the corresponding gravitational potential is calculated by solving Poisson's equation. In this paper, the case is revised as we solve the modified Poisson's equation. The simulations are also not scale-free, which was possible for some Newtonian galaxy models.

(2)A full orbital library is established. In other words, it is necessary to generate a large set of initial conditions for the orbits.

(3) The whole system is divided into many cells with the same mass. The fraction of time spent by each orbit in each cell $O_{i j}$ is recorded into a large matrix.

(4)The non-negative occupation numbers $W_{j}$ are programmed in a linear equation group with the above matrix and the desired mass in each cell. The weights are then solved by non-negative least squares inversion. Note that the relation between orbital weights and the density distribution is linear independent of gravity theories.

The linear equation group can be written as 


$$
\sum_{j=1}^{N_{o}} W_{j} O_{i j}=M_{i}, \quad i=1, \ldots . ., N_{c},
$$

where $M_{i}$ is the mass of each grid cell, $N_{o}$ is the number of orbits, here we have $N_{o}=6840$. $N_{c}$ is the number of cells, and we adopt $N_{c}=912 . W_{j} \geq 0$ is the weight number of the occupation. We follow MF96 to divide the first octant into 960 cells, which can be described by the following steps. The galaxy was divided by 20 ellipsoidal shells into 21 sections of equal mass. For each shell, only the first octant was considered. The first octant was then divided into three parts by the planes $z=h_{z} x / h_{x}, y=h_{y} x / h_{x}$, and $z=h_{z} y / h_{y}$. Each part, for example the first part, which contained the x-axis, was divided by $h_{x} y / h_{y} x=1 / 5,2 / 5,2 / 3$ and $h_{x} z / h_{z} x=1 / 5,2 / 5,2 / 3$ into 16 cells. The total number of cells is $20 \times 3 \times 16=960$. We consider the inner 912 cells in solving the linear equation groups.

Generally, equation (7) can be solved by linear programming (e.g., Schwarzschild 1979, 1982, 1993) using Lucy's method (Lucy 1974; Statler 1987), or maximum entropy methods (e.g., Richstone \& Tremaine 1988; Statler 1991; Gebhardt et al. 2003), or with the least squares solver (Lawson \& Hanson 1974)(MF96; van de Ven et al. 2006; Capuzzo-Dolcetta et al. 2006). The four methods have their own advantages and disadvantages, which we do not discuss here. We adopt the least squares method to solve the linear programming. Then the minimum square deviation can be written as

$$
\chi^{2}=\frac{1}{N_{c}} \sum_{j=1}^{N_{c}}\left(C_{i}-\sum_{i=1}^{N_{o}} w_{j} O_{i j}\right)^{2}
$$

For the symmetry of the triaxial model, only one octant $O_{i j}$ is considered in solving the linear programming.

\section{Conclusion and discussion}

In practice, a parameter $\delta$ is adopted to describe the departure form self-consistency, which is defined as in MF96:

$$
\delta=\frac{\sqrt{\chi^{2}}}{\bar{M}},
$$

where $\bar{M}$ is the average mass in each cell. Figure 4 shows the departure from self-consistency as a function of the number of orbits. Note that there is a remarkably strong dependence on the number of orbits. The departure parameter $\delta$ drops quickly when the number of orbits exceeds $4000 . \delta$ is $10^{-15}$ when the 6840 orbits are adopted in the optimization routine, 
which shows that all three triaxial models are self-consistent in MOND. Amazingly, we also find that the departure parameter $\delta$ is insensitive to $a_{0}$. In other words, if one of the triaxial galaxies is self-consistent in MOND, then the same model with a different value of $a_{0}$ will also be self-consistent.

Figure 5 shows the contribution of mass for various orbital families in the self-consistent model. Chaotic orbits contribute a larger fraction of mass with the increase of energy. This shows a certain degree of consistency with that of Capuzzo-Dolcetta et al. (2007). However, discrepancies between our results and that of Capuzzo-Dolcetta et al. (2007) are apparent. There is a clear variational trend of the cumulative energy distribution of the various orbital families in Newton's case, however, no apparent variational trend for different orbits has been found in MOND.

In Figure6, we show the radial profile of the rescaled radial velocity dispersion $\sigma_{r} / V_{\text {cir }, \infty}$ (left panel) and the radial profile of the anisotropy parameter $\beta=1-0.5\left(<V^{2}-V_{r}^{2}>\right) /<V_{r}^{2}>$ (right panel), where $V_{\text {cir, } \infty}$ is the circular velocity at infinity, and $V_{r}$ is radial velocity. The parameter $V^{2}$ is defined as $V^{2}=v_{x}^{2}+v_{y}^{2}+v_{z}^{2}$, where $v_{x}, v_{y}$ and $v_{z}$ are the three components of velocity. It can be seen that the profiles of the radial velocity dispersion are nearly flat in MOND, except in the central region. Recently, Capuzzo-Dolcetta et al. (2007) studied the same model as that used here, but in the Newtonian gravity. It can be seen that the profile of the velocity dispersion in our model shows a certain degree of consistency with that of Capuzzo-Dolcetta et al. (2007), but the discrepancies between our results and that of Capuzzo-Dolcetta et al. (2007) are apparent. There is central peak in our velocity dispersion profile. The global average of the velocity $V_{\mathrm{rms}}$ is $170.49,78.84$ and $42.70 \mathrm{~km} / \mathrm{s}$ for $a_{0}=0.083,0.833$, and 8.333, respectively (see the last column of Table 1).

The anisotropy parameter $\beta \geq 0$ suggests that the stars have high radial velocity and the ratio of $\left\langle V_{r}^{2}>/<V^{2}-V_{r}^{2}>\right.$ reaches a maximum value at $2-3 \mathrm{kpc}$. Our anisotropy profiles are similar to that of Nipoti et al. (2007b) form dissapationless collapse in MOND (their figur 2) and in Newtonian gravity van Albada (1982) and Wang et al. (2008a). A gently rising beta is widely used (e.g., Milgrom \& Sanders 2003). However, the peak in the anisotropy profile in our model is not seen in any simulations and observations to our best knowledge. This is might be indication of problem of MOND. Our models have a sudden change of the amount of box orbit near shells 10-12 (radius 2-3kpc), which could be the reason.

In order to check the stability of our models, we used the Antonov's third law (Binney $\&$ Tremaine 1987). If the stellar system is stable, then its density $\rho$ and potential $\Phi$ satisfy everywhere the inequality $\mathrm{d}^{3} \rho_{b} / \mathrm{d} \Phi^{3}<0$. Figure 7 shows that $\mathrm{d}^{3} \rho_{b}(0, y, 0) / \mathrm{d}|\Phi(0, y, 0)|^{3}$ is positive everywhere along the intermediate axis for our three models, since the values of 
potential are negative, our models are consistent with the law, which means the models appear globally stable. We will also be checking the stability of the models in collaboration with groups which have MOND N-body code. Effects due to pattern rotation and external field in MOND remain to be studied (Wang et al. 2008b).

We have used the Schwarzschild approach to examine whether the triaxial galaxies are self-consistent in MOND. Using the Bologna Poisson solver to determine the potential and the accelerations at some discrete points, we used three-dimensional interpolation then obtain the potential and accelerations at arbitrary point. The orbits conserve energy to a very high level of accuracy, which shows that our method is feasible in calculating orbits using a grid-based MONDian potential. The departure parameter $\delta$ shows that the triaxial galaxy models adopted in this paper in MOND are self-consistent. Our results show that it is theoretically allowed to have triaxial galaxies in rigorous equilibrium in a universe with either non-Newtonian gravity or certain models of Dark Energy (Zhao 2007).

We thank the referee for the constructive comments which improved our presentation, Luca Ciotti, Pasquale Londrio, Carlo Nipoti for generously sharing their MONDian Poisson solver code and Dr. Scott Gregory for assistance on written English, Zuhui Fan for discussions. YGW is supported by the National Science Foundation of China under grants 10525314, 10533010, by the Chinese Academy of Sciences under grant KJCX3-SYW-N2, and by the Ministry of Science and Technology under the national basic sciences program (973) under grant 2007CB815401. HZ acknowledges partial support of PPARC Advanced Fellowship and National Natural Science Foundation of China (NSFC under grant 10428308). XW acknowledges the support of SUPA studentship.

\section{REFERENCES}

Angus, G. W., Shan, H. Y., Zhao, H. S., \& Famaey, B. 2007, ApJ, 654, L13

Angus, G. W., Famaey, B., Tiret, O., Combes, F., \& Zhao, H. S. 2008, MNRAS, 383, L1

Bekenstein, J. 2004, Phys. Rev. D, 70, 083509

Bekenstein, J., \& Milgrom, M. 1984, ApJ, 286, 7

Binney, J. 2005, MNRAS, 363, 937

Binney, J., \& Tremaine, S. 1987, Galactic Dynamics (Princeton: Princeton Univ. Press)

Capuzzo-Dolcetta, R., Leccese, L., Merritt, D., \& Vicari, A. 2007, ApJ, 666, 165 
Chen, D. M., \& Zhao, H. S., 2006, ApJ, 650, 9

Ciotti, L., Londrillo, P., \& Nipoti, C. 2006, ApJ, 640, 741

Famaey, B., Bruneton., J. P., \& Zhao, H. S. 2007, MNRAS, 377, L79

Fehlberg, E. 1968, Classical Fifth-, Sixth-, Seventh-, and Eighth-Order Rung-Kutta Formullas with Stepsize Control (NASA Tech. Rep. R-287; Washington: NASA)

Gebhardt, K., et al. 2003, ApJ, 583, 92

Gentile, G., Salucci, P., Klein, U., \& Granato, G. L. 2007, MNRAS, 375, 199

Gentile, G. et al., 2007, A\&A, 472, 25

Halle, A., \& Zhao H. S. 2007, arXiv:0711.0958

Hernquist, L. 1990, ApJ, 356, 359

Kuijken, K. 2004, ASPC, 317, 310

Lawson, C. L., \& Hanson, R. 1974, Solving Least Squares Problems (2d ed.; Englewood Cliffs: Prentice Hall)

Lee, J., \& Suto, Y. 2003, ApJ, 585, 151

Lucy, L. B. 1974, AJ, 79, 745

McGaugh, S. S., de Blok, W. J. G., Schombert, J. M., Kuzio de Naray, R., \& Kim, J. H. 2007, ApJ, 659, 149

Merritt, D., \& Fridman, T. 1996, ApJ, 460, 136 (MF96)

Milgrom, M., \& Sanders, R. H. 2003, ApJ, 599, L25

Milgrom, M. 1983, ApJ, 270, 365

Nipoti, C., Londrillo, P., Zhao, H. S., \& Ciotti, L. 2007a, MNRAS, 379, 597

Nipoti, C., Londrillo, P., \& Ciotti, L. 2007b, ApJ, 660, 256

Richstone, D. O., \& Tremaine, S. 1988, ApJ, 327, 82

Rix, H. W., et al. 1997, ApJ, 488, 702

Sanders, R. H., \& Noordermeer, E. 2007, MNRAS, 379, 702 
Sarkar, S. 2007, arXiv0710.5307

Schwarzschild, M. 1993, ApJ, 409, 563

Schwarzschild, M. 1982, ApJ, 263, 599

Schwarzschild, M. 1979, ApJ, 232, 236

Shan, H. Y., Feix, M., Famaey, B., Zhao, H. S. 2008, MNRAS, submitted

Skordis, C., Mota, D. F., Ferreira, P. G., \& Boehm, C. 2006, Phys.Rev.Lett. 96, 011301

Statler, T. S. 1991, AJ, 102, 882

Statler, T. S. 1987, ApJ, 321, 113

Tiret, O., \& Combes, F. 2007, A\&A, 464, 517

van Albada, T. S. 1982, MNRAS, 201, 939

van der Marel, R. P., et al. 1998, ApJ, 493, 613

van de Ven, G., van den Bosch, R. C. E., Verolme, E. K., \& de Zeeuw, P. T. 2006, A\&A, 445,513

Wang, Y. -G., et al., 2008a, in preparation

Wang, Y. -G., et al., 2008b, in preparation

Wang, Y. -G., \& Fan, Z. -H. 2004, ApJ, 617, 847

White, S. D. M. 2007, RPPh, 70, 883

Wu, X., et al., 2007, ApJ, 665, L101

Wu, X., et al., 2008, MNRAS submitted

Zhao, H. S. 1996, MNRAS, 283, 149

Zhao, H. S. 2006, astro-ph/0610056

Zhao, H. S. 2007, ApJ, 671, 1

Zhao, H. S., Bacon, D. J., Taylor, A. N., \& Horne, K. 2006, MNRAS, 368, 171

Zhao, H. S., \& Famaey, B. 2006, ApJ, 638, L9 
Zhao, H. S., \& Tian, L. A\&A, 2006, 450, 1005

Zhao, H. S., Xu, B. X., \& Dobbs, C. 2008, MNRAS submitted

This preprint was prepared with the AAS LATEX macros v5.2.

Table 1: Main physical parameters for our three ellipsoidal MOND models

\begin{tabular}{lllll}
\hline \hline Model & $a_{0}^{a}[\mathrm{~km} / \mathrm{s} / \mathrm{Gyr}]$ & $M\left[M_{\odot}\right]$ & $V_{\text {cir }, \infty}[\mathrm{km} / \mathrm{s}]$ & $V_{\mathrm{rms}}[\mathrm{km} / \mathrm{s}]$ \\
\hline$a_{0}=0.083$ & 3600 & $10^{11}$ & 198.6 & 170.5 \\
$a_{0}=0.833$ & 3600 & $10^{10}$ & 111.7 & 78.8 \\
$a_{0}=8.333$ & 3600 & $10^{9}$ & 62.8 & 42.7 \\
\hline
\end{tabular}

${ }^{a}$ real value of $a_{0}$. 


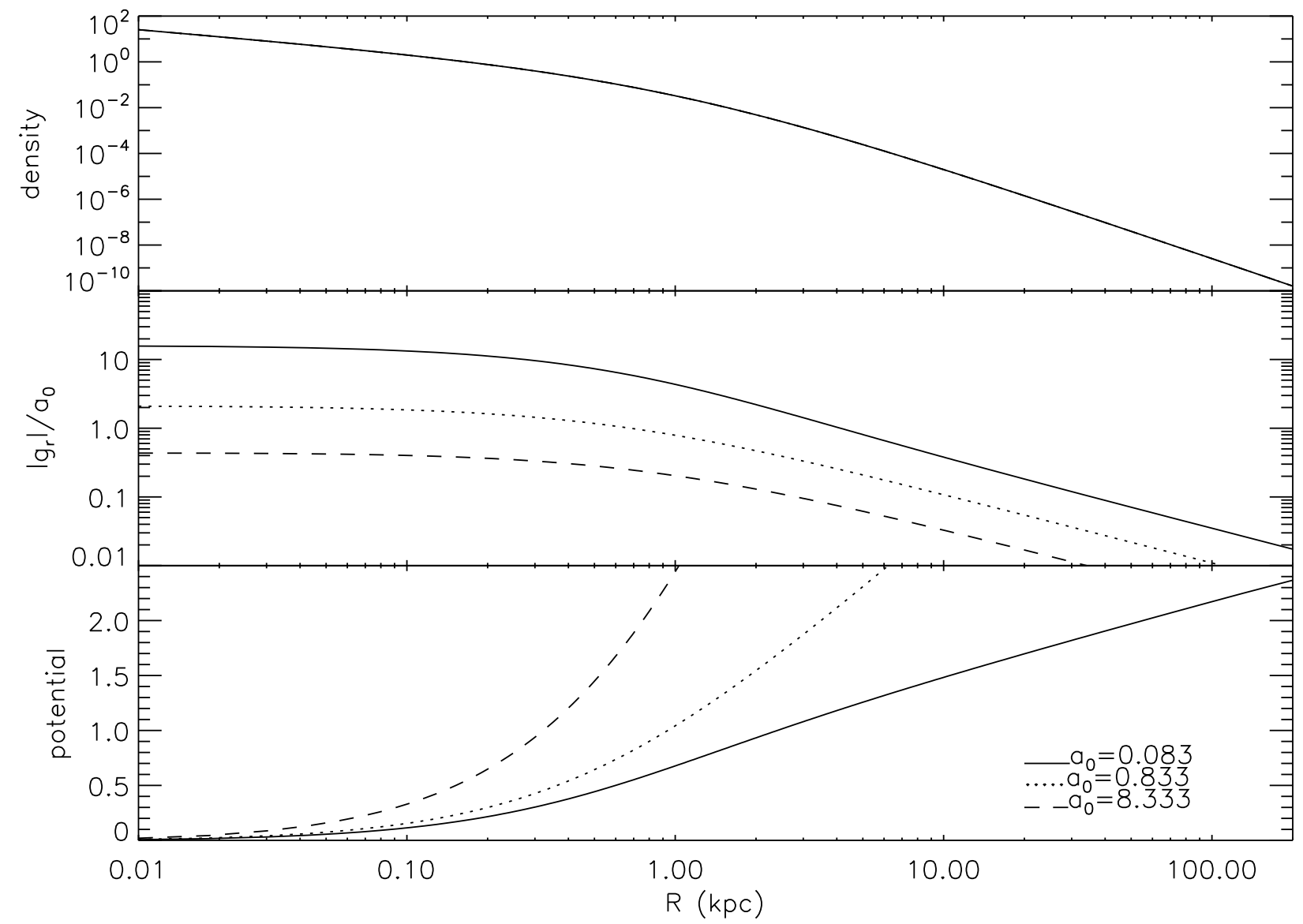

Fig. 1.- Upper: the radial distribution of the dimensionless density $\rho$ in units of $M /\left(2 \pi h_{x} h_{y} h_{z}\right)$, which is identical for the three models. Middle: the MOND gravity strength $\left|g_{r}\right| / a_{0}$ as a function of $R$ along the long axis. Lower: the potential major axis profile, for different values of $a_{0}$, obtained from the Poisson solver. 

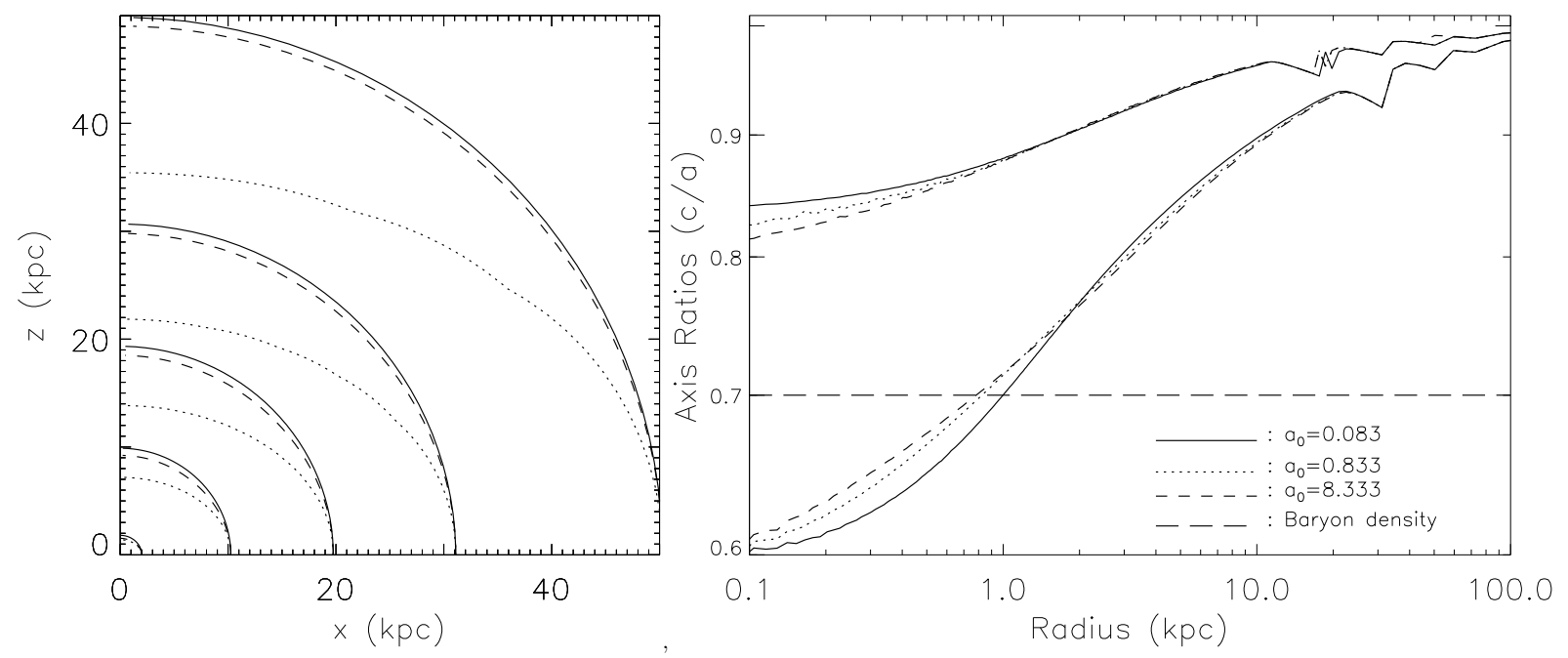

Fig. 2.- Left panels: Isopotentials (solid lines), isodensities of effective dark matter(dashed) and isodensities of baryons (dotted) on the x-z plane, in the model of $a_{0}=0.833$. The contours of the other two models are almost same. The isopotentials are essentially a snapshot of the metric perturbations, and the isodensities of effective DM halos are images of the perturbation of certain Dark Energy fluid (Zhao 2007). Right panel shows the axis ratio $c / a$ (the ratio between the short and long axis) of the potentials (upper series of curves), effective DM densities (lower series of curves) and baryon densities(the long-dashed line) in a log-log space. For each series, the solid, dotted and dashed lines are for different models of $a_{0}$ as in the legend. The oscillations at large radii are due to the numerical resolution. 

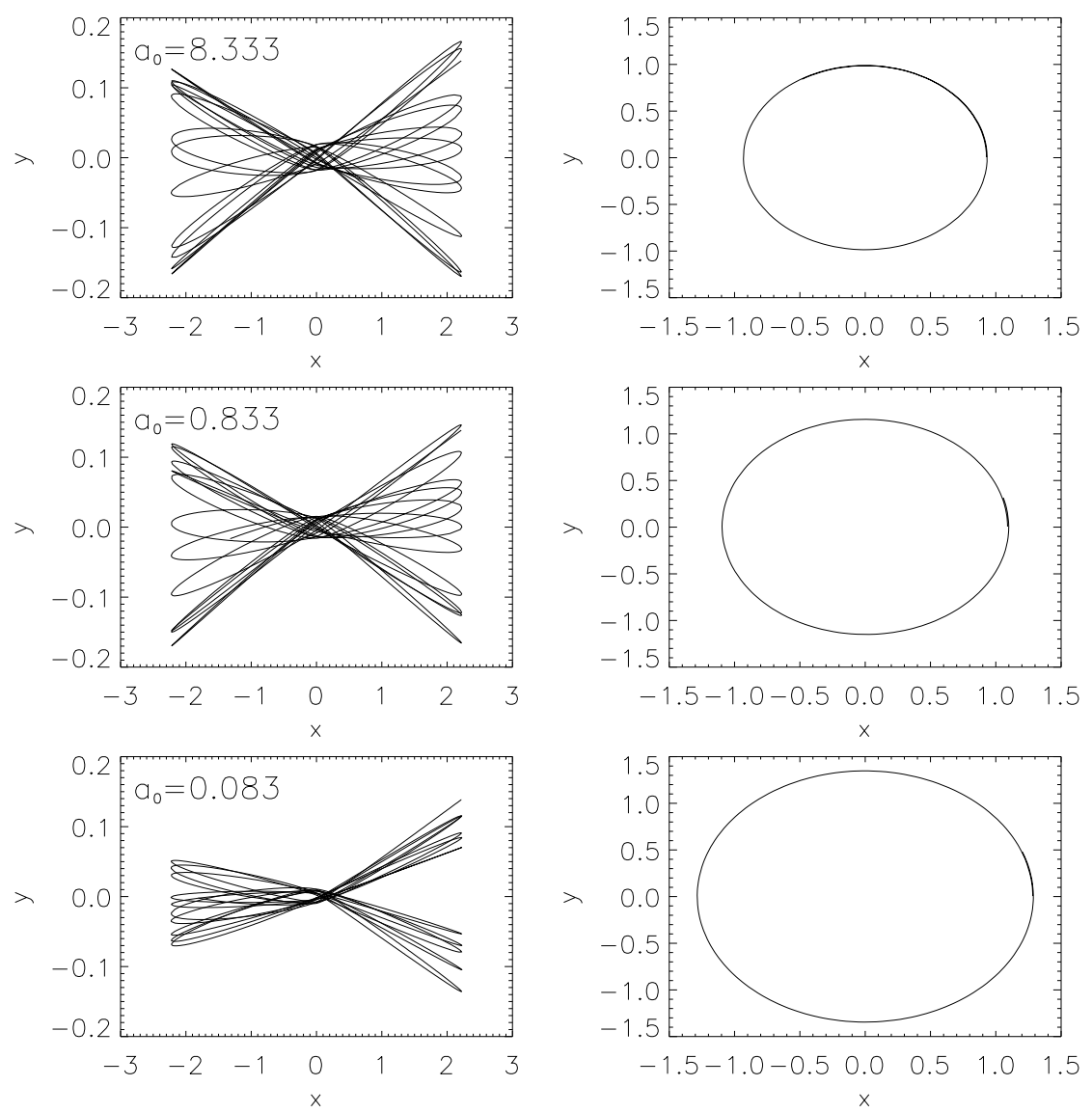

Fig. 3.- Orbits in a nonrotating triaxial potential in MOND. The left panels are for a stationary start space, while the right panels are for a $\mathrm{x}-\mathrm{z}$ start space (1:1 resonant orbit). The energy is from the 10th cell. 


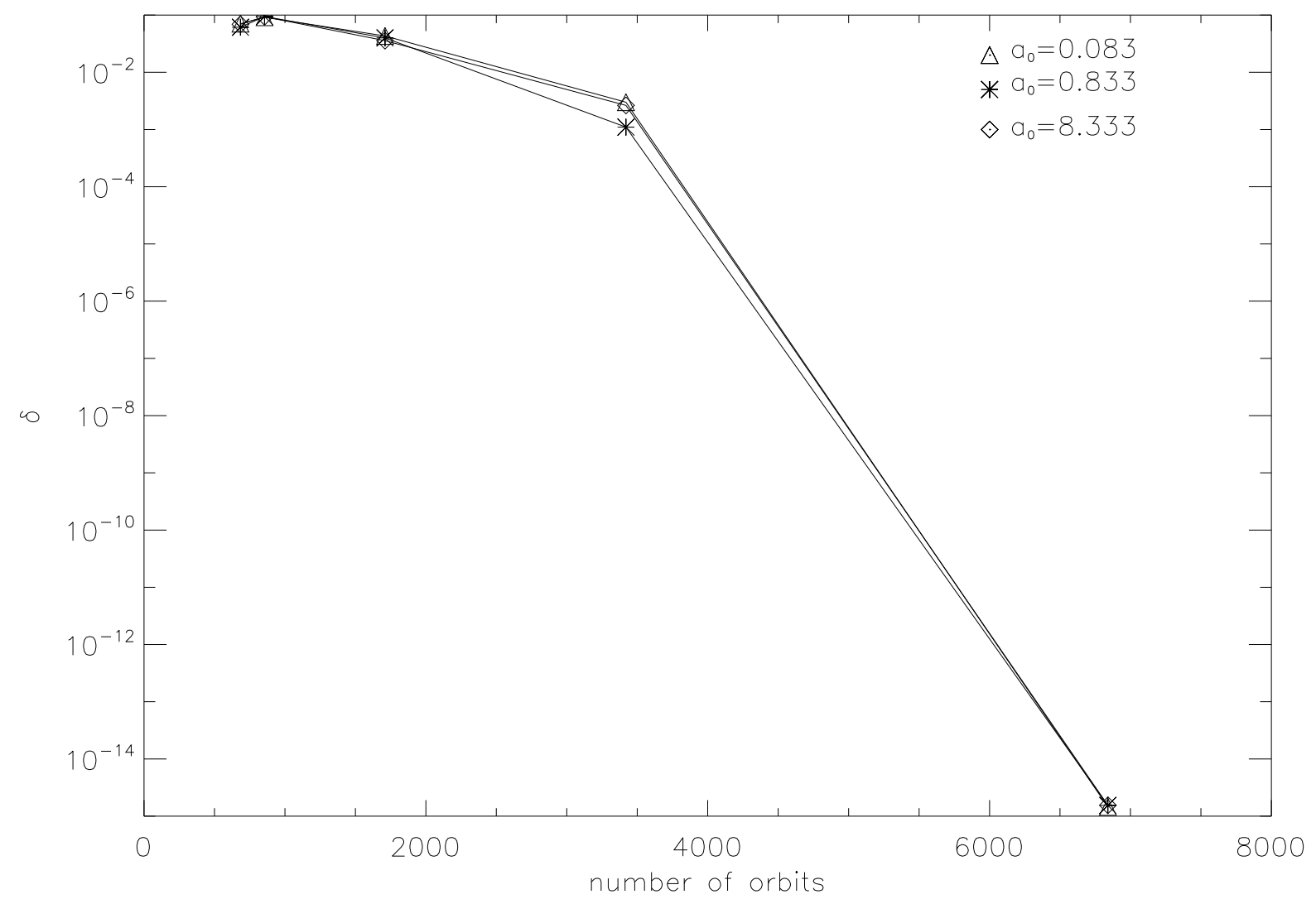

Fig. 4.- Departure form self-consistency $\delta$ as a function of the number of orbits for different values of $a_{0}$. The triangles, asterisks, and diamonds represent $a_{0}=0.083,0.833$, and 8.333, respectively. 
Table 2: The energy and dynamical time in every shell

\begin{tabular}{llllllll}
\hline \hline & \multicolumn{3}{l}{ Radius $^{a}$} & \multicolumn{3}{c}{ Energy } & \multicolumn{3}{l}{$T_{D}$} \\
\cline { 2 - 7 } Shell & $a_{0}{ }^{b}$ & $a_{0}=0.083$ & $a_{0}=0.833$ & $a_{0}=8.333$ & $a_{0}=0.083$ & $a_{0}=0.833$ & $a_{0}=8.333$ \\
\hline 1 & 0.2791 & 0.2933 & 0.4113 & 0.9006 & 2.640 & 2.200 & 1.477 \\
2 & 0.4464 & 0.4163 & 0.5990 & 1.341 & 3.600 & 2.955 & 1.960 \\
3 & 0.6076 & 0.5120 & 0.7536 & 1.713 & 4.485 & 3.645 & 2.395 \\
4 & 0.7744 & 0.5940 & 0.8908 & 2.055 & 5.400 & 4.335 & 2.815 \\
5 & 0.9529 & 0.6675 & 1.021 & 2.384 & 6.400 & 5.065 & 3.255 \\
6 & 1.148 & 0.7355 & 1.145 & 2.706 & 7.470 & 5.815 & 3.720 \\
7 & 1.366 & 0.7995 & 1.267 & 3.030 & 8.685 & 6.705 & 4.255 \\
8 & 1.612 & 0.8610 & 1.390 & 3.358 & 10.16 & 7.530 & 4.810 \\
9 & 1.896 & 0.9209 & 1.513 & 3.695 & 11.71 & 8.770 & 5.490 \\
10 & 2.226 & 0.9800 & 1.639 & 4.046 & 13.71 & 10.05 & 6.220 \\
11 & 2.620 & 1.039 & 1.771 & 4.416 & 16.04 & 11.56 & 7.120 \\
12 & 3.097 & 1.099 & 1.910 & 4.810 & 19.00 & 13.47 & 8.185 \\
13 & 3.690 & 1.160 & 2.057 & 5.237 & 22.72 & 15.80 & 9.510 \\
14 & 4.449 & 1.225 & 2.217 & 5.705 & 27.68 & 18.76 & 11.23 \\
15 & 5.458 & 1.294 & 2.395 & 6.231 & 34.23 & 22.71 & 13.64 \\
16 & 6.866 & 1.370 & 2.597 & 6.834 & 44.00 & 28.59 & 16.76 \\
17 & 8.974 & 1.456 & 2.835 & 7.554 & 58.23 & 39.75 & 21.31 \\
18 & 12.48 & 1.560 & 3.131 & 8.459 & 82.40 & 50.47 & 29.12 \\
19 & 19.49 & 1.697 & 3.533 & 9.702 & 131.2 & 78.07 & 44.72 \\
20 & 40.49 & 1.915 & 4.196 & 11.77 & 279.0 & 161.6 & 91.36 \\
\hline
\end{tabular}

${ }^{a}$ along the x-axis.

${ }^{b} a_{0}=0.083,0.833,8.333$. 


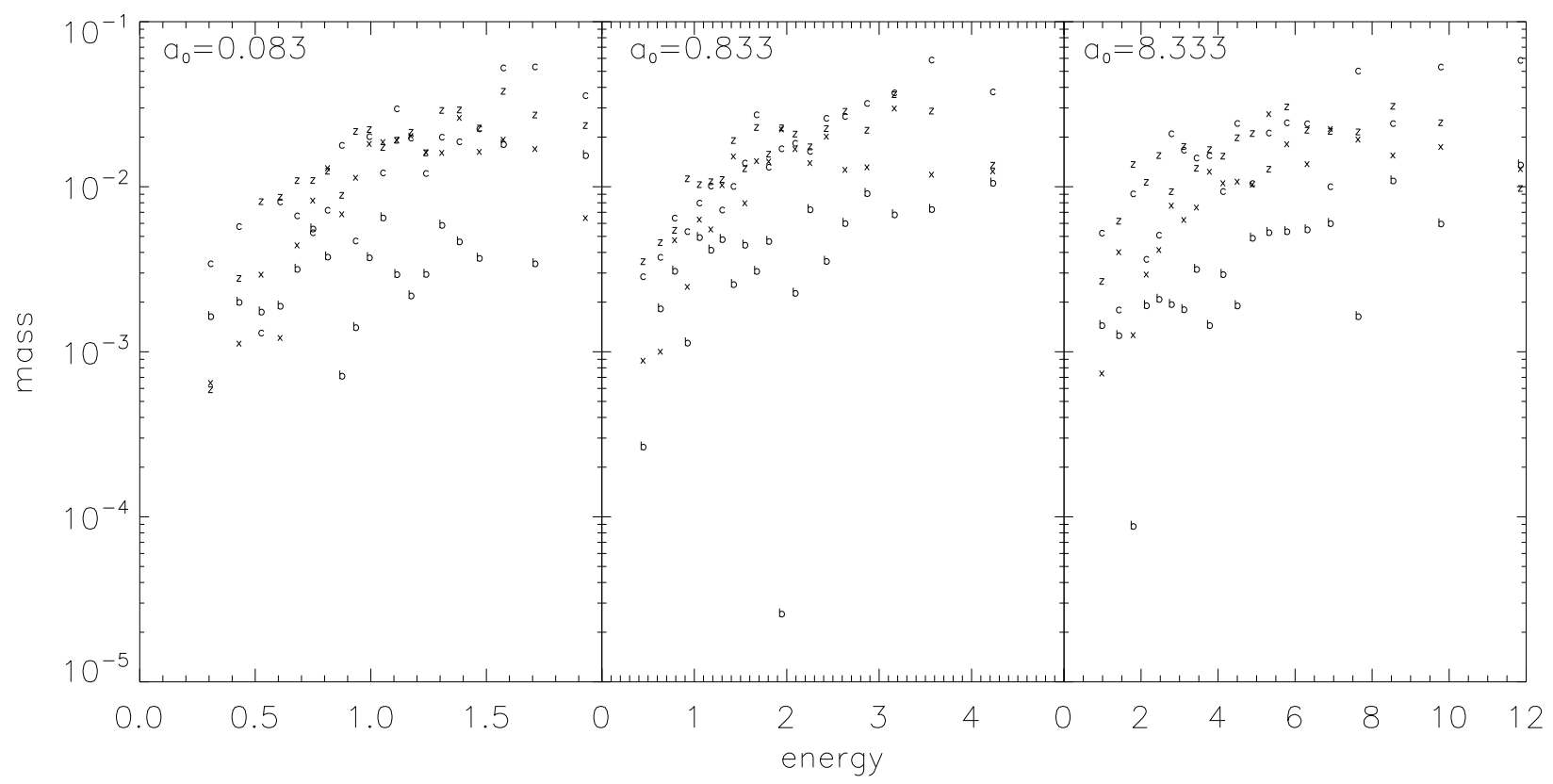

Fig. 5.- Orbital families in the self-consistent models. The symbols b, x, z, and c denote the mass contributed by box, x-tube, z-tube, and chaotic orbits, respectively.
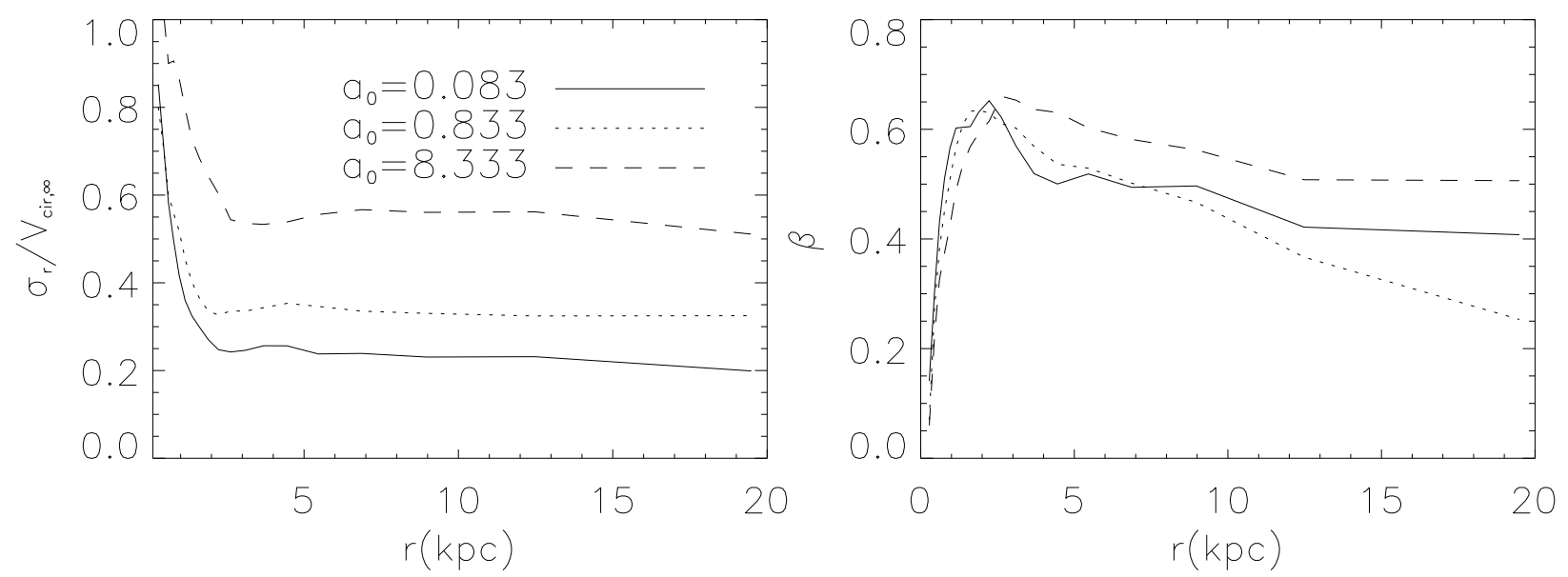

Fig. 6. - The radial profiles of the rescaled radial velocity dispersion $\sigma_{r} / V_{\text {cir, } \infty}$ (left panel) and of the anisotropy parameter $\beta=1-0.5<V^{2}-V_{r}^{2}>/<V_{r}^{2}>$ (right panel). The solid, dotted and dashed lines represent $a_{0}=0.083,0.833,8.333$, respectively. 


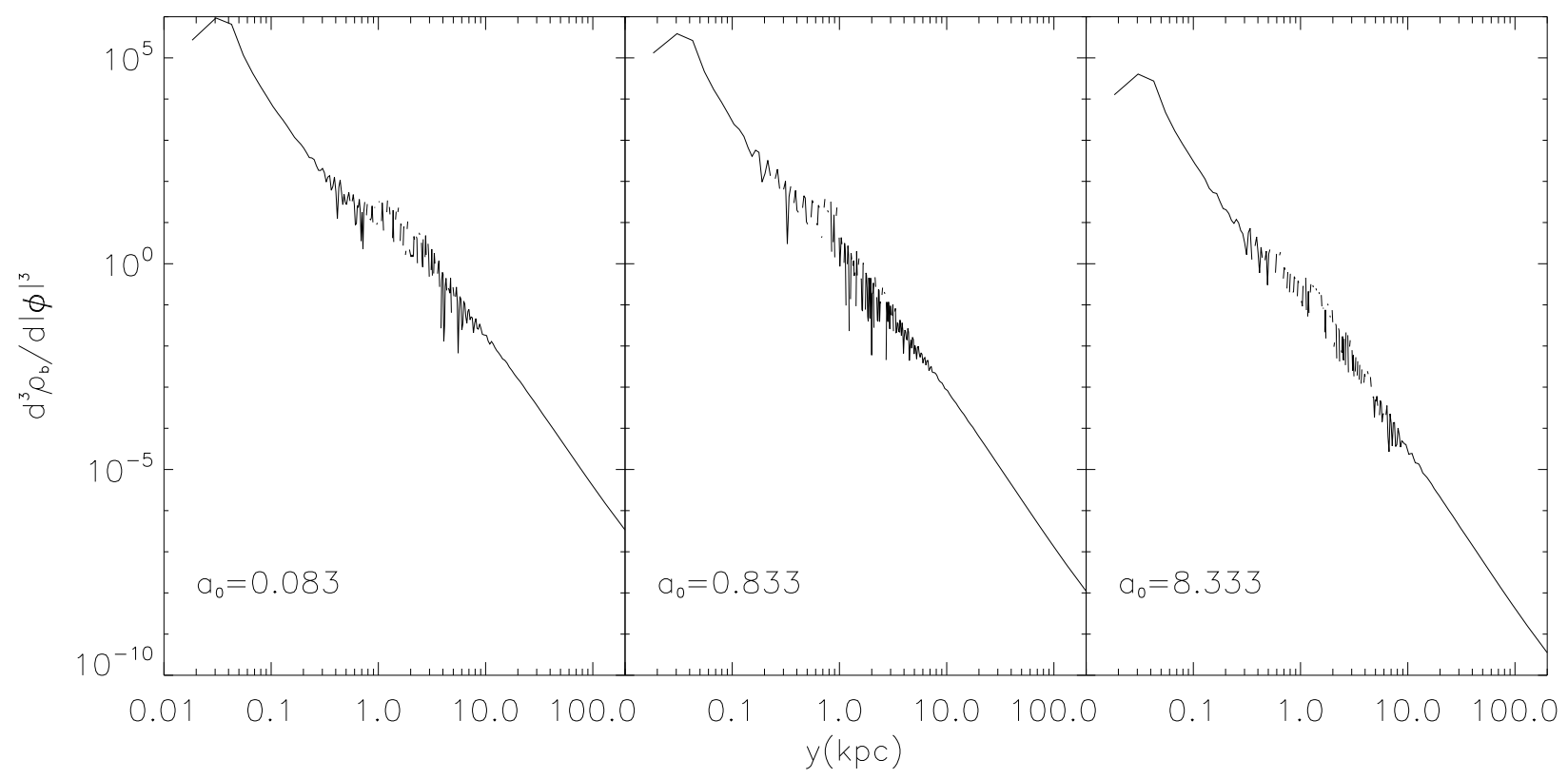

Fig. 7.- Distribution of $\mathrm{d}^{3} \rho_{b}(0, y, 0) / \mathrm{d}|\Phi(0, y, 0)|^{3}$ as function of MOND potential $\mid \Phi(0, y, 0 \mid)$ along the intermediate axis for our three models. 